\title{
Blade-Directed Totally Laparoscopic Feeding Jejunostomy - How to do it
}

\author{
Chun Chih Lai, Chun Yi Tsai* and Chun Nan Yeh \\ Department of General Surgery, Chang Gung Memorial Hospital, Taiwan
}

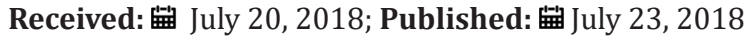

*Corresponding author: Chun Yi Tsai, Assistant professor, Department of General Surgery, Chang Gung Memorial Hospital, Linkou branch, Taiwan,Chang Gung University, No.5, Fu Shing Street, Kweishan District, Taoyuan City, 333, Taiwan.

\begin{abstract}
Jejunostomy is the recommended route for enteral nutrition in certain patients. Various methods and equipment had been proposed to complete the operation laparoscopically. The method we proposed is based on the triangular rule of trocar orientation with typically available tube and equipment. It minimized the wounds to one $11 \mathrm{~mm}$ trocar for the laparoscope plus two $5 \mathrm{~mm}$ trocars for intracorporeal suturing, by creating the jejunostoma with straightforward surgical blade penetration. Sixty-nine patients underwent the operation as either a solitary procedure or a part of the operation based on preoperative indications. The majority of the patients received feeding within 3 day after operation (84\%) without major complications. Fifteen patients experienced tube dislodgement or occlusion during the follow up but did not encounter difficulty while exchanging new tubes. The method we proposed could be done with readily available equipment and minimized wounds and did not yield major complications.

Keywords: Laparoscopic Feeding Jejunostomy; Minimally Invasive Surgery; Enteral Nutrition
\end{abstract}

\section{Introduction}

Enteral nutrition routes exhibit advantages over parenteral routes. Based on the clinical scenario, enteral feeding can be provided through gastrostomy or jejunostomy either endoscopically or surgically generated. However, debate remains regarding the choice of enterostomy, and jejunostomy is preferred to gastrostomy in certain conditions, particularly when the stomach is to be reserved for subsequent reconstruction. Since the first reported totally laparoscopic feeding jejunostomy [1], various methods had been proposed with acceptable results [2-5]. These methods require either specific equipment or modified tubes that increased excessive cost. Small wound is a major advantage of laparoscopic operation compared with conventional laparotomy, particularly when treating malnourished patients who are at risk of impaired wound healing. We make every effort to minimize the wounds. Our proposed method is based on the triangle rule of orientation of trocars and the revision of conventional totally laparoscopic feeding jejunostomy. We then create the insertion site of the tube by straightforward penetration with surgical blade under laparoscopy. After each fixation of peritonization, the needle could be extracted via the $5 \mathrm{~mm}$ trocars. With the above two maneuvers, totally laparoscopic feeding jejunostomy could be completed with one $11 \mathrm{~mm}$ trocar plus two $5 \mathrm{~mm}$ trocars by intracorporeal sutures with typically available equipment.

\section{Materials and Methods}

The patients were placed in the Trendelenburg position at $30^{\circ}$ and tilted to the left side. The surgeon stood at the right cephalic side of the patient, and the assistant stood at the right caudal side. We applied a $11 \mathrm{~mm}$ trocar through the umbilicus for the laparoscope. Then the two $5 \mathrm{~mm}$ working ports were created: one is at the lower midline $10 \mathrm{~cm}$ from the umbilicus and the other is at the left subcostal area $10 \mathrm{~cm}$ from the umbilicus (Figure 1). The orientation of the trocars and the presumed jejunostoma conformed to the "triangle rule", ensuring a smooth operation.

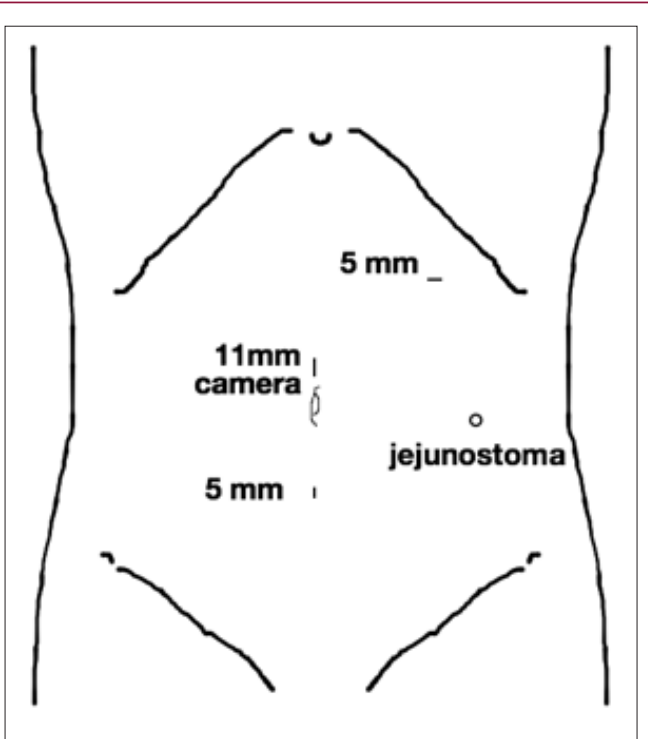

Figure 1: Position and size of the trocars and the jejunostoma. 


\section{Operative Steps}

After identifying the ligament of Treitz, we anchored the desired jejunum (20 cm distal to the ligament) to the peritoneum at the presumed jejunostoma position as the first peritonization stitch. The jejunotomy was created by electrocauterizing hook after the surrounding purse-string suture was completed. A No.11 surgical blade was applied at the skin at the presumed jejunostoma position, and penetrated into the peritoneal cavity under laparoscopic direction (Figure 2). The generated tract accommodated the passage of the No.16 nasogastric (NG) tube, which was guided through the jejunotomy into the distal bowel lumen until reaching the desired length (Figure 3). The purse-string suture was tightened. Finally, we completed the peritonization stitches in the remaining three quadrants to surround the jejunotomy. The fluency of the tube was tested at desufflation status before the trocars were removed. We used disposable silk (Ethicon Perma-Hand Silk Suture, ETHICON, LLC, USA) for the sutures. These were inserted into the peritoneal cavity through the $11 \mathrm{~mm}$ video port, and could be extracted through the $5 \mathrm{~mm}$ port easily in parallel direction.

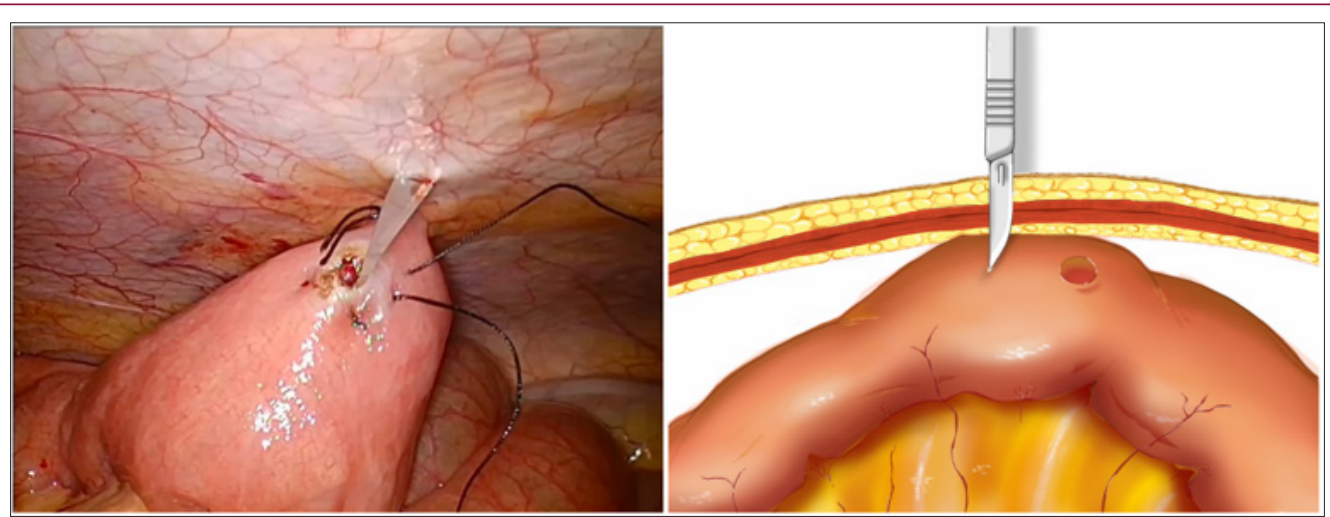

Figure 2: Whole layer penetration of abdominal wall by the surgical blade (scalpel).
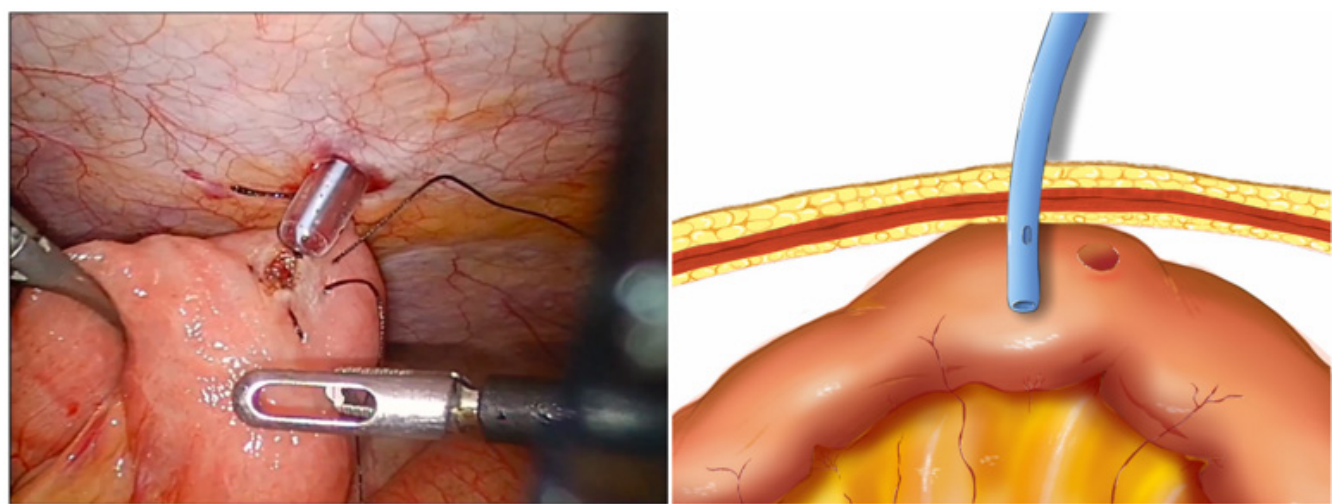

Figure 3: Apply NG tube through the blade-created tract into the jejunum.

\section{Results}

Table 1: Indications for feeding jejunostomy.

\begin{tabular}{|c|c|}
\hline Diagnosis & Number \\
\hline Esophageal/ Gastric cancer & 49 \\
\hline Head/ Neck neoplasms & 10 \\
\hline Repeated aspiration pneumonia & 6 \\
\hline Lung cancer associated sequelae & 3 \\
\hline Perforated gastric ulcer* & 1 \\
\hline
\end{tabular}

*Feeding jejunostomy was performed during the primary operation.

From August 2011 to May 2012 and July 2013 to June 2017, a single surgeon performed the operation on 69 patients. The indications were summarized in Table 1. Five patients received feeding jejunostomy as an adjunctive procedure during a primary operation: four of the patients exhibited unresectable gastric cancer from the cardia to the body with peritoneal carcinomatosis; the other one was a malnourished, aged patient who exhibited a large perforated peptic ulcer. Most patients started feeding via the jejunostomy tube within 3 days following the operation (58/69, $84 \%$ ), and none of them exhibited leakage, tube dysfunction, or surgical mortality. Two patients (3\%) experienced pain and erythematous changes around the jejunostoma, which resolved after conservative treatment with parenteral antibiotics. Fifteen of the patients (22\%) experienced either tube dislodgement or occlusion during follow-up; no difficulties were encountered when changing to new feeding tubes (Fr.16 NG tubes). Five of the patients had their tubes removed after treatment for primary disease and did not experience complications. The remaining 64 patients did not encounter remarkable extravasation of the enteral content until the date of expire due to disease progression or the date of follow up (December 31, 2017), regardless of whether the tube was changed or not. The operation time ranged from 144 minutes at the 
very first patient to 37 minutes for the shortest one (mean time was 68 minutes), and it demonstrated a decreasing trend following each operation (Figure 4).

\section{Discussion}

Minimally invasive procedures have become one of the standard in abdominal surgeries since the introduction of the laparoscope. Surgeons throughout the world have established standard laparoscopic procedures in nearly all fields; however, no unified method has been proposed for conducting laparoscopic feeding jejunostomies [2]. Since intracorporeal suturing is an essential skill for laparoscopic surgeons, we attempt to simplify totally laparoscopic feeding jejunostomy into a procedure that requires only repeated intracorporeal sutures ( 5 sutures including the purse string suture on the jejunum). Figure 4 disclosed that the operation time decreased following each operation stemming from more proficient intracorporeal sutures. The proposed operation could be performed using readily available NG tubes, surgical blades, and standard laparoscopic equipment. Jimenez et al. [5] proposed the trocar-guided method to pass the tube into the peritoneal cavity. We replaced this step by using direct penetration with a surgical blade to limit the costs of trocars and ensure a tight caliber tract for the tube. This procedure could be done with a minimal expense of equipment. Second, a tight contact between the tract and tube prevents air leakage, facilitating the passage of the tube until the operation is completed. Malnutrition is a risk factor associated with surgical wound complications [6]. Most of the patients requiring feeding enterostomy for feeding were malnourished. We speculate that a smaller wound yields a correspondingly smaller possibility of wound dehiscence. To achieve the goal of minimized wounds on abdominal wall, our proposed method involves extracting the needle in parallel direction directly through the $5 \mathrm{~mm}$ port based on the flexible nature of needles, enabling the operation to be completed using two $5 \mathrm{~mm}$ trocars.

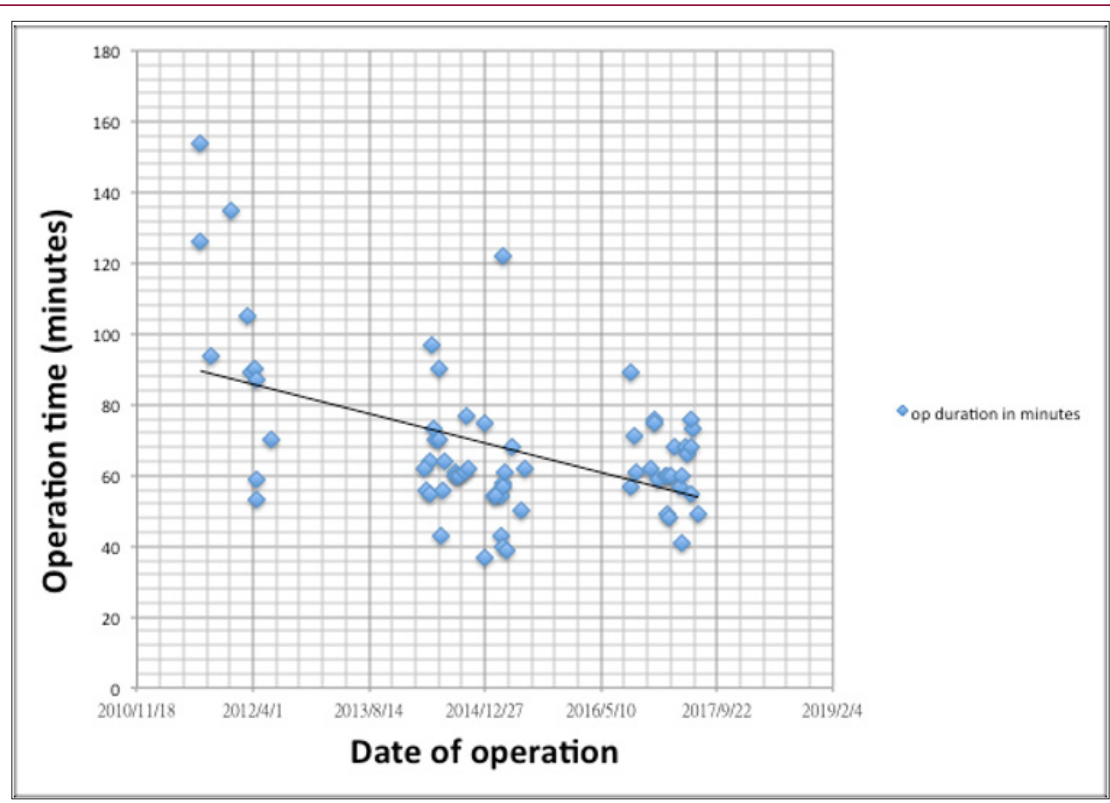

Figure 4: Operation time of each patient.

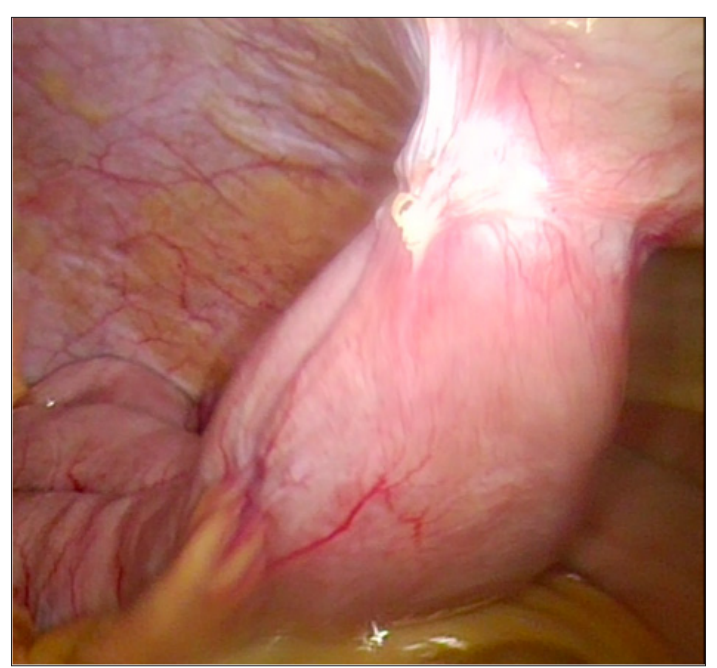

Figure 5: Intraperitoneal view of the jejunostoma $14^{\text {th }}$ weeks after the first operation. 
Occlusion or dislodgement of tube are encountered randomly owing to feeding formula or care givers. We did not experience any difficulty in removing the occluded tubes or inserting new tubes for the 15 patients with accidental tubes. The skin exit site remained in close contact with the new Fr.16 NG tube, exhibiting no extravasation of bowel content after feeding or changing tubes. T-tubes or T-fasteners were applied in several series, but there was no statement about the changing of tubes. One of our patients with advanced esophageal cancer had tube slippage 14 weeks after operation, and re-insertion was failed due to completely healed cutaneous wound when he visited our hospital 20 hours after the event. We performed exactly the same procedure again and the in vivo image disclosed solid healing of previously created stoma (Figure 5). In summary, we proposed our method in performing totally laparoscopic feeding jejunostomy with minimal wounds and readily available equipment, which yields acceptable safety at the same time.

\section{Acknowledgement}

We appreciate all the nurses and nutrition therapists in caring these patients wholeheartedly.

ISSN: 2574-1241

DOI: 10.26717/BJSTR.2018.07.001457

Chun Yi Tsai. Biomed J Sci \& Tech Res

(C) (i) This work is licensed under Creative Submission Link: https://biomedres.us/submit-manuscript.php

\section{References}

1. Allen JW, Ali A, Wo J, Bumpous JM, Cacchione RN (2002) Totally laparoscopic feeding jejunostomy. Surg Endosc 16(12):1802-1805.

2. Han-Geurts IJ, Lim A, Stijnen T, Bonjer HJ (2005) Laparoscopic feeding jejunostomy: a systematic review. Surg Endosc 19(7): 951-957.

3. Senkal M, Koch J, Hummel T, Zumtobel V (2004) Laparoscopic needle catheter jejunostomy: modification of the technique and outcome results. Surg Endosc 18(2): 307-309.

4. Mohiuddin SS, Anderson CE (2011) A novel application for singleincision laparoscopic surgery (SILS): SIL jejunostomy feeding tube placement. Surg Endosc 25(1): 323-327.

5. Jimenez Rodriguez RM, Lee MR, Pigazzi A (2012) Trocar guided laparoscopic feeding jejunostomy: a simple new technique. Surg Laparosc Endosc Percutan Tech 22(5): e250-253.

6. Wild T, Rahbarnia A, Kellner M, Sobotka L, Eberlein T (2010) Basics in nutrition and wound healing. Nutrition 26(9): 862-866.

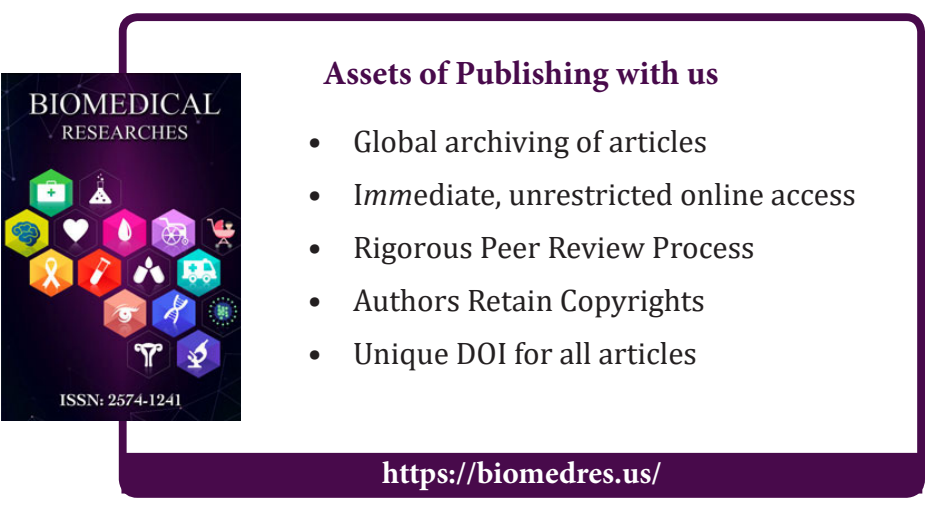

